\title{
GLOBAL UNIQUENESS AND LIPSCHITZ STABILITY OF RESIDUAL STRESS FROM ONE BOUNDARY MEASUREMENT
}

\author{
V. ISAKOV ${ }^{1}$ AND N. KIM ${ }^{1}$
}

\begin{abstract}
In this paper, we consider an elasticity system with residual stress. We are interested in recovery of the residual stress from one boundary measurement. We obtain uniqueness and the (best possible) Lipschitz stability results without any smallness assumption.
\end{abstract}

\section{INTRODUCTION}

We consider an elasticity system with residual stress $R$. Under reasonable conditions this system is time hyperbolic and hence the initial boundary value problem for it is well posed in standard energy spaces. We are interested in recovery of the residual stress from additional boundary data. This system is anisotropic, which creates a challenge for its identification: there are only recent results [9], [10] when $R$ is small. The goal of this paper is to obtain uniqueness and the (best possible) Lipschitz stability results without any smallness assumption. The residual stress model is of significant and growing interest for material science (i.g. for steel production), for geophysics, and more recently for elastic prospecting of human body. We let $x \in \mathbb{R}^{3}$ and $(x, t) \in \Omega \subset \mathbb{R}^{4}$. The residual stress is modeled by a symmetric second-rank tensor $R(x)=\left(r_{j k}(x)\right)_{j, k=1}^{3} \in \mathcal{C}^{2}(\bar{\Omega})$ which is divergence free $\nabla \cdot R=0$ [13]. Let $\mathbf{u}(x, t)=\left(u_{1}, u_{2}, u_{3}\right)^{\top}: \Omega \rightarrow \mathbb{R}^{3}$ be the displacement vector in $\Omega$. We introduce the operator of the linear elasticity with the residual stress

$$
\begin{gathered}
\mathbf{A}_{R} \mathbf{u}= \\
\rho \partial_{t}^{2} \mathbf{u}-\mu \Delta \mathbf{u}-(\lambda+\mu) \nabla(\operatorname{div} \mathbf{u})-\nabla \lambda \operatorname{div} \mathbf{u}-2 \epsilon(\mathbf{u}) \nabla \mu-\operatorname{div}((\nabla \mathbf{u}) R),
\end{gathered}
$$

where $\rho \in \mathcal{C}^{1}(\bar{\Omega}), \lambda, \mu \in \mathcal{C}^{2}(\bar{\Omega})$ are density and Lamé parameters depending only on $x, \epsilon(\mathbf{u})=\frac{1}{2}\left(\partial_{i} u_{j}+\partial_{j} u_{i}\right)$.

To state the main result we need the pseudo-convexity conditions with respect to the general partial differential operator of second order

$$
A=\sum_{j, k=1}^{n} a^{j k} \partial_{j} \partial_{k}+\sum b^{j} \partial_{j}+c
$$

in a bounded domain $\Omega$ of the space $\mathbb{R}^{n}$ with the real-valued coefficients $a^{j k} \in \mathcal{C}^{1}(\bar{\Omega}), b^{j}, c \in L^{\infty}(\Omega)$. The principal symbol of this operator is

$$
A(x ; \zeta)=\sum a^{j k}(x) \zeta_{j} \zeta_{k} .
$$

We use the following convention and notations. Sums are over repeated indices $j, k, l, m=1, \ldots, n$. Let $\partial_{j}=$ $\partial / \partial x_{j}, \partial=\left(\partial_{1}, \ldots, \partial_{n}\right), D=-i \partial, \alpha=\left(\alpha_{1}, \ldots, \alpha_{n}\right)$ is a multi-index with integer components, $\zeta^{\alpha}=\zeta_{1}^{\alpha_{1}} \cdots \zeta_{n}^{\alpha_{n}}$, $D^{\alpha}$ and $\partial^{\alpha}$ are defined similarly.

\footnotetext{
${ }^{1}$ Department of Mathematics and Statistics, Wichita State University, Wichita, KS 67260-0033, USA; e-mail: isakov@wichita.edu
}

(c) EDP Sciences, SMAI 2009 
A function $\psi$ is called pseudo-convex on $\bar{\Omega}$ with respect to $A$ if $\psi \in \mathcal{C}^{2}(\bar{\Omega}), A(x, \nabla \psi(x)) \neq 0, x \in \bar{\Omega}$, and

$$
\begin{gathered}
\sum \partial_{j} \partial_{k} \psi(x) \frac{\partial A}{\partial \zeta_{j}} \frac{\partial A}{\partial \zeta_{k}}(x ; \xi)+ \\
\sum\left(\frac{\partial A}{\partial \zeta_{k}} \partial_{k} \frac{\partial A}{\partial \zeta_{j}}-\partial_{k} A \frac{\partial^{2} A}{\partial \zeta_{j} \partial \zeta_{k}}\right) \partial_{j} \psi(x ; \xi) \geq K|\xi|^{2}
\end{gathered}
$$

for some positive constant $K$, for any $\xi \in \mathbb{R}^{n}$ and any point $x$ of $\bar{\Omega}$, provided

$$
A(x ; \xi)=0, \sum \frac{\partial A}{\partial \zeta_{j}}(x, \xi) \partial_{j} \psi(x)=0 .
$$

Let $\square(\mu ; R)=\partial_{t}^{2}-\sum_{j k} \frac{\mu \delta_{j k}+r_{j k}}{\rho} \partial_{j} \partial_{k} . \quad \nu$ is the outward normal to the boundary of a domain, $C, \gamma$ are generic constants (different at different places) depending only on the operators $\mathbf{A}_{R}, \mathbf{A}_{R(2)}$ (but not on $\mathbf{A}_{R(1)}$, on the upper bound of the norms $\left\|r_{j k}\right\|_{C^{6}(\bar{G})}$, on the ellipticity constant of $\mathbf{A}_{R(1)}$, on the initial data $\mathbf{u}_{0}, \mathbf{u}_{1}$, the boundary data $\mathbf{g}_{0}$, on the function $\psi$, and on the domain $\Omega$. Any additional dependence will be indicated. We recall that

$$
\|u\|_{(k)}(\Omega)=\left(\sum_{|\alpha| \leq k} \int_{\Omega}\left|\partial^{\alpha} u\right|^{2}\right)^{\frac{1}{2}}
$$

is the norm in the Sobolev space $H_{(k)}(\Omega)$ and \|\|$_{2}=\|\|_{(0)}$ is the $L^{2}$-norm.

We will use the weight function

$$
\varphi=e^{\gamma \psi} \text {. }
$$

We have

Theorem 1.1. Let $\psi$ be pseudo-convex with respect to $\square(\mu ; R) ; \square(\lambda+2 \mu ; R)$ in $\bar{\Omega}$. Then there are constants $C, \gamma \in(0,1)$ such that

$$
\begin{aligned}
& \int_{\Omega}\left(\tau\left(\left|\nabla_{x, t} \mathbf{u}\right|^{2}+\left|\nabla_{x, t} \operatorname{div} \mathbf{u}\right|^{2}+\left|\nabla_{x, t} \operatorname{curl} \mathbf{u}\right|^{2}\right)\right. \\
& \left.+\tau^{3}\left(|\mathbf{u}|^{2}+|\operatorname{div} \mathbf{u}|^{2}+\left|\operatorname{curl}_{\mathbf{u}}\right|^{2}\right)\right) e^{2 \tau \varphi} \\
& \leq C \int_{\Omega}\left(\left|\mathbf{A}_{R} \mathbf{u}\right|^{2}+\left|\nabla\left(\mathbf{A}_{R} \mathbf{u}\right)\right|^{2}\right) e^{2 \tau \varphi}
\end{aligned}
$$

for all $\mathbf{u} \in H_{(3)}^{0}(\Omega)$ and $C<\tau$.

In [8] this result was obtained when $R$ is "small". The second large parameter was introduced by one of the authors around 1985 [5] and used then to obtain first uniqueness of the continuation results for the classical elasticity system and later on for the thermoelasticity system [4]. In [6] we obtained Carleman estimates with two large parameters for general scalar second order operators $A$ and derived from these estimates Theorem 1.1 .

Now we state results about identification of residual stress from additional boundary data. In Theorems 1.2, $1.3 \Omega=G \times(-T, T)$ where $G$ is a bounded domain in $\mathbb{R}^{3}$ with $C^{8}$-boundary and $\Gamma \subset \partial G \times(-T, T)$.

Let $\mathbf{u}(; 1), \mathbf{u}(; 2)$ be solutions to

$$
\begin{gathered}
\mathbf{A}_{R} \mathbf{u}=\mathbf{0} \text { in } \Omega, \\
\mathbf{u}=\mathbf{u}_{0}, \partial_{t} \mathbf{u}=\mathbf{u}_{1} \text { on } G \times\{0\}, \mathbf{u}=\mathbf{g}_{0} \text { on } \partial G \times(-T, T),
\end{gathered}
$$

corresponding to sets of coefficients $R(; 1)$ and $R(; 2)$, respectively. We will assume that $\mathbf{u}_{0} \in H^{9}(G), \mathbf{u}_{1} \in$ $H^{8}(G), \mathbf{g}_{0} \in C^{9}(\partial G \times[-T, T])$. Then our conditions on the coefficients of residual stress systems and on $G$ by known energy estimates (like in [3], [9]) imply that

$$
\left\|\partial_{x}^{\alpha} \partial_{t}^{\beta} \mathbf{u}\right\|_{\infty}(\Omega) \leq C, \text { when }|\alpha| \leq 2, \beta \leq 5 .
$$


We can consider the boundary stress data as measurements (observations). We introduce the norm of the differences of the lateral Cauchy data

$$
F=\sum_{\beta=2}^{4}\left\|\partial_{t}^{\beta} \partial_{\nu}(\mathbf{u}(; 2)-\mathbf{u}(; 1))\right\|_{\left(\frac{3}{2}\right)}(\Gamma)
$$

By examining the equation (1.1), we can see that since residual stress tensor is divergence free it appears in the equation without first derivatives. It turns out that a single set of Cauchy data is sufficient to recover the symmetric (variable) matrix $R$. To guarantee the uniqueness, we impose some non-degeneracy condition on the initial data $\left(\mathbf{u}_{0}, \mathbf{u}_{1}\right)$. Let

$$
\mathbf{M}=\left(\begin{array}{llllll}
\partial_{1}^{2} \mathbf{u}_{0} & 2 \partial_{1} \partial_{2} \mathbf{u}_{0} & 2 \partial_{1} \partial_{3} \mathbf{u}_{0} & \partial_{2}^{2} \mathbf{u}_{0} & 2 \partial_{2} \partial_{3} \mathbf{u}_{0} & \partial_{3}^{2} \mathbf{u}_{0} \\
\partial_{1}^{2} \mathbf{u}_{1} & 2 \partial_{1} \partial_{2} \mathbf{u}_{1} & 2 \partial_{1} \partial_{3} \mathbf{u}_{1} & \partial_{2}^{2} \mathbf{u}_{1} & 2 \partial_{2} \partial_{3} \mathbf{u}_{1} & \partial_{3}^{2} \mathbf{u}_{1}
\end{array}\right)
$$

Note that $\mathbf{M}(x)$ is a $6 \times 6$ matrix-valued function. We assume that

$$
\operatorname{det} \mathbf{M}>\varepsilon_{0}>0 \text { on } \Omega \text {. }
$$

One can check that, for example, $\mathbf{u}_{0}(x)=\left(x_{1}^{2}, x_{2}^{2}, x_{3}^{2}\right)^{\top}$ and $\mathbf{u}_{1}(x)=\left(x_{2} x_{3}, x_{1} x_{3}, x_{1} x_{2}\right)^{\top}$ satisfy $(1.10)$ with $\varepsilon_{0}=2^{6}$.

We first state the Hölder type estimate of determining coefficients in $\Omega(\varepsilon)$ defined as $\Omega \cap\{\psi>\varepsilon\}$.

Theorem 1.2. Assume that $\psi$ is pseudo-convex with respect to $\square(\mu ; R(; 2)), \square(\lambda+2 \mu ; R(; 2))$ in $\bar{\Omega}$. Let the initial data $\left(\mathbf{u}_{0}, \mathbf{u}_{1}\right)$, satisfy (1.10). Assume that $\bar{\Omega}(0) \subset \Omega \cap \Gamma$. Then there exist constants $C, \gamma \in(0,1)$ such that

$$
\|R(; 2)-R(; 1)\|_{(1)}(\Omega(\varepsilon)) \leq C F^{\gamma} .
$$

This result is derived from Theorem 1.1 in [7] by combining Theorem 1.1 and methods of [8].

If $\Gamma$ is the whole lateral boundary and $T$ is sufficiently large, then a much stronger (and in a certain sense best possible) Lipschitz stability estimate holds.

In Theorem 1.3 we assume that the system (1.1) is t-hyperbolic. A sufficient condition is

$$
0 \leq \lambda, 0<2 \mu I_{3}+R \text { on } \bar{G} \text {. }
$$

This condition is satisfied when any eigenvalue of the matrix $R$ is strictly greater than $-2 \mu$, this happens when, for example, $\sum_{i, j=1}^{3} r_{i j}^{2}<4 \mu^{2}$ on $\bar{\Omega}$.

Theorem 1.3. Let $\psi$ be pseudo-convex with respect to $\square(\mu ; R(; 2), \square(\lambda+2 \mu ; R(; 2))$ in $\bar{\Omega}$. Assume that

$$
\psi<0 \text { on } \bar{G} \times\{-T, T\}, 0<\psi \text { on } G \times\{0\}
$$

Let the initial data $\left(\mathbf{u}_{0}, \mathbf{u}_{1}\right)$ satisfy (1.10). Let $\Gamma=\partial G \times(-T, T)$.

Then there exist $C$ such that for $R(; 1), R(; 2)$ satisfying the condition

$$
R(; 1)=R(; 2) \quad \text { on } \quad \Gamma,
$$

one has

$$
\|R(; 2)-R(; 1)\|_{(1)}(\Omega) \leq C F,
$$

where $C$ are depending only on the operator $A_{R(; 2)}$ and on the function $\psi$.

This paper is devoted to a proof of Theorem 1.3.

We observe that the condition (1.10) is quite restrictive for many applications. However, recently new measurements (in particular by using interior sources) become available and feasible in important geophysical and medical applications (e.g. [1]). The nondeneracy conditions, like (1.10), are getting important since they explain what positions and intensities of sources are needed for stable reconstruction of coefficients of partial differential equations modeling corresponding physical phenomenae. 


\section{Pseudo-COnVExity CONDitions}

Explicit and clear pseudo-convexity conditions on $\psi(x, t)=|x-\beta|^{2}-\theta^{2} t^{2}$ for a isotropic hyperbolic operator of second order are given in [5], section 3.4. The general case is much more difficult to handle. In [11] for a special anisotropic operator $A$ it is shown that $\psi(x, t)=x_{n}-\theta^{2} t^{2}$ is pseudo-convex with respect to $A$ if the speed of propagation is monotone in the $x_{n^{-}}$direction. According to [12] $\psi(x, t)=d^{2}(x, a)-\theta^{2} t^{2}(d$ is the distance in the Riemannian metric determined by the elliptic part of $A$ ) is pseudo-convex if sectional curvatures are nonpositive. We give explicit pseudo-convexity conditions for $\psi(x, t)=|x-\beta|^{2}-\theta^{2} t^{2}$ in the most general case. Also we show that explicit smallness condition on $R$ (compared to nonexplicit conditions in [8], [9]) suffices for pseudo-convexity. Smallness of residual stress is a natural assumption for applications to geophysics and material science. Observe that our choice of $\psi$ is geometrically motivated (by Eucledean metric). Indeed, it is most natural choice for inverse problems when the metric generated by $A$ is not known.

We denote by $\|R\|$ the norm $\left(\sum_{j, k=1}^{3} r_{j k}^{2}\right)^{\frac{1}{2}}$ of a matrix $R=\left(r_{j k}\right)$. Let $D=\sup |x-\beta|$ over $x \in G$ and $d=\inf |x-\beta|$ over $x \in G$.

Lemma 2.1. Let $\theta, s$ be some numbers and $\beta \in \mathbb{R}^{n}$. Let $\rho, \lambda, \mu$ be constants, the matrix $R$ be symmetric positive at any point of $\Omega$ and

$$
2 \mu \rho \theta^{2}+3\|R(x)+\mu I\|\|\nabla R(x)\||x-\beta|<2 \mu^{2} \text { when } x \in \bar{G}
$$

Let

$$
\theta^{2} T^{2}<d^{2}
$$

Then the function $\psi(x, t)=|x-\beta|^{2}-\theta^{2} t^{2}-d^{2}$ is pseudo-convex with respect to the anisotropic wave operator $A=\square(\mu ; R)$ in $\bar{\Omega} \cap\left\{|x-\beta|^{2}>\theta^{2} t^{2}\right\}$.

Proof uses standard calculations to verify pseudo-convexity condition (1.2) and is given in [6].

Assume that

$$
D^{2}<2 d^{2}
$$

Let

$$
\frac{D^{2}-d^{2}}{\theta^{2}}<T^{2}<\frac{d^{2}}{\theta^{2}}
$$

Then the conditions (2.2), (2.3) are satisfied. So if in addition (2.1) holds we have the conclusion of Theorem 1.3. The condition (2.3) can be always achieved by choosing $\beta$ at some distance from $G$. So condition of Theorem 1.3 are satisfied when observation time is sufficiently large and residual stress is relatively small.

Now for a general second order operator we give explicit pseudo-convexity conditions of $\psi(x, t)=|x-\beta|^{2}-$ $\theta^{2} t^{2}, x, \beta=\left(0, \ldots, 0, \beta_{n}\right) \in \mathbb{R}^{n}$ when $\beta_{n}$ is large enough, not assuming that $R$ is small. In Lemma $2.2 G$ is a domain in $\mathbb{R}^{n}$ and $\Omega=G \times(-T, T)$.

We introduce the coefficient matrix

$$
M_{A}=\left(\begin{array}{cccc}
a_{11} & a_{12} & \ldots & a_{1 n} \\
a_{21} & a_{22} & \ldots & a_{2 n} \\
\ldots & \ldots & \ldots & \ldots \\
a_{n 1} & a_{n 2} & \ldots & a_{n n}
\end{array}\right)
$$

and the linear differential operator $\left(M_{n} \cdot \nabla\right)=a_{n 1} \partial_{1}+a_{n 2} \partial_{2}+\cdots+a_{n n} \partial_{n}$.

Lemma 2.2. Let

$$
A(x, \partial)=\partial_{t}^{2}-\sum_{j, k=1}^{n} a_{j k} \partial_{j} \partial_{k}
$$


where $a_{j k}(x) \in \mathcal{C}^{1}(\bar{G})$ and

$$
\sum_{j, k=1}^{n} a_{j k} \xi_{j} \xi_{k} \geq \varepsilon_{0}|\xi|^{2}
$$

for some $\varepsilon_{0}>0$ and all $\xi \in \mathbb{R}^{n}$. Let

$$
\psi(x, t)=|x-\beta|^{2}-\theta^{2} t^{2}, \quad \beta=\left(0, \ldots, 0, \beta_{n}\right) .
$$

Assume that

$$
\xi^{\top}\left(M_{n} \cdot \nabla\right) M \xi \geq \varepsilon_{0}|\xi|^{2} \text { for any } \xi \in \mathbb{R}^{n}, \text { for some } \varepsilon_{0}>0,
$$

and that one of the following conditions is satisfied: (1) the last column (or row) vector valued function $M_{n}$ of matrix $M$ only depends on $x_{n}$, or (2) $n=2$ and $a_{22} \partial_{1} a_{12}-a_{12} \partial_{1} a_{22} \leq 0$ on $G$.

Then there is large $\beta_{n}$ such that the function $\psi$ is pseudo-convex with respect to $A$ in $\bar{\Omega}$.

\section{Proof of Lemma $\mathbf{2 . 2}$}

To check the pseudo-convexity condition (1.2) we let $t=x_{0}, A(x, \xi)=\xi_{0}^{2}-\sum_{j, k=1}^{n} a_{j k} \xi_{j} \xi_{k}$,

$$
\mathcal{H}_{1}=\sum_{j, k=0}^{n} \partial_{j} \partial_{k} \psi \frac{\partial A}{\partial \xi_{j}} \frac{\partial A}{\partial \xi_{k}}
$$

and

$$
\mathcal{H}_{2}=\sum_{j, k=0}^{n}\left(\left(\partial_{k} \frac{\partial A}{\partial \xi_{j}}\right) \frac{\partial A}{\partial \xi_{k}}-\partial_{k} A \frac{\partial^{2} A}{\partial \xi_{j} \partial \xi_{k}}\right) \partial_{j} \psi
$$

Using the first condition $\xi_{0}^{2}=\sum_{j, k=1}^{n} a_{j k} \xi_{j} \xi_{k}$ of $(1.3)$, we yield

$$
\mathcal{H}_{1}=-8 \theta^{2} \sum_{j, k=1}^{n} a_{j k} \xi_{j} \xi_{k}+8 \sum_{j=1}^{n}\left(\sum_{k=1}^{n} a_{j k} \xi_{k}\right)^{2}
$$

Since $\partial_{0} A, \partial_{0} \frac{\partial A}{\partial \xi_{0}}, \frac{\partial^{2} A}{\partial \xi_{j} \partial \xi_{0}}, \partial_{k} \frac{\partial A}{\partial \xi_{0}}, \frac{\partial^{2} A}{\partial \xi_{0} \partial \xi_{k}}, j, k=1, \ldots, n$ are all zeros, and since $\partial_{j} \psi=2\left(x_{j}-\beta_{j}\right), j=$ $1, \ldots, n, \beta=\left(0, \ldots, 0, \beta_{n}\right)$,

$$
\begin{gathered}
\mathcal{H}_{2}=\sum_{j, k=1}^{n}\left\{\left(-2 \sum_{l=1}^{n} \partial_{k} a_{j l} \xi_{l}\right)\left(-2 \sum_{m=1}^{n} a_{m k} \xi_{m}\right)\right. \\
\left.-\left(-\sum_{p, q=1}^{n} \partial_{k} a_{p q} \xi_{p} \xi_{q}\right)\left(-2 a_{j k}\right)\right\} 2\left(x_{j}-\beta_{j}\right) .
\end{gathered}
$$

To achieve pseudo-convexity of $\psi$ we need positivity of $\mathcal{H}_{1}+\mathcal{H}_{2}$ provided (1.3) holds. To do so we we collect the (dominating) terms of $\mathcal{H}_{1}+\mathcal{H}_{2}$ with large factor $\beta_{n}$ in

$$
\mathcal{H}_{3}(\xi)=4 \beta_{n} \sum_{j, l=1}^{n} \sum_{k=1}^{n}\left\{a_{n k} \partial_{k} a_{j l}-2 a_{l k} \partial_{k} a_{j n}\right\} \xi_{j} \xi_{l}
$$

¿From the second condition (1.3),

$$
\sum_{j=0}^{n} \frac{\partial A}{\partial \xi_{j}} \partial_{j} \psi=-4 \theta^{2} t \xi_{0}+2 \sum_{j, k=1}^{n} a_{j k} \xi_{k} x_{j}-2 \sum_{k=1}^{n} a_{n k} \xi_{k} \beta_{n}=0
$$


so

$$
\sum_{k=1}^{n} a_{n k} \xi_{k}=\frac{1}{\beta_{n}} 0(|\xi|) .
$$

where we also used (2.4).

Consider the quadratic polynomial (2.6) by using (2.7):

$$
\begin{gathered}
\mathcal{H}_{3}(\xi)=4 \beta_{n}\left(\sum_{j, k, l=1}^{n} a_{n k} \partial_{k} a_{j l} \xi_{j} \xi_{l}\right. \\
\left.\left.-2 \sum_{k=1}^{n-1} \sum_{l=1}^{n} a_{l k} \xi_{l}\left(\sum_{j=1}^{n} \partial_{k} a_{j n} \xi_{j}\right)\right)-2 \sum_{l=1}^{n} a_{l n} \xi_{l}\left(\sum_{j=1}^{n} \partial_{n} a_{j n} \xi_{j}\right)\right)= \\
4 \beta_{n}\left(\sum_{j, k, l=1}^{n} a_{n k} \partial_{k} a_{j l} \xi_{j} \xi_{l}-2 \sum_{k=1}^{n-1} \sum_{j, l=1}^{n} a_{l k} \partial_{k} a_{j n} \xi_{j} \xi_{l}\right)+\ldots
\end{gathered}
$$

where ... denotes terms bounded by $C|\xi|^{2}$.

Finally, it turns out that

$$
\mathcal{H}_{3}(\xi)=4 \beta_{n}\left(\xi^{\top}\left(M_{n} \cdot \nabla\right) M \xi-2 \xi^{\top}(M \cdot \nabla) M_{n}^{\top} \xi\right)+\ldots
$$

where

$$
\begin{gathered}
\xi^{\top}\left(M_{n} \cdot \nabla\right) M \xi-2 \xi^{\top}(M \cdot \nabla) M_{n}^{\top} \xi= \\
\left(\sum_{j, k, l=1}^{n} a_{n k} \partial_{k} a_{j l} \xi_{j} \xi_{l}-2 \sum_{k=1}^{n-1} \sum_{j, l=1}^{n} a_{l k} \partial_{k} a_{j n} \xi_{j} \xi_{l}\right) .
\end{gathered}
$$

In the case (1) (2.8) implies the positivity of (1.2), because the second sum is zero.

When $n=2, \xi_{2}=-\frac{a_{21}}{a_{22}} \xi_{1}+\frac{1}{\beta_{2}} 0(|\xi|)$, so from $(2.7)$,

$$
\begin{gathered}
\sum_{k=1}^{1} \sum_{j, l=1}^{2} a_{l k} \partial_{k} a_{j 2} \xi_{j} \xi_{l} \\
=\left(a_{11} \partial_{1} a_{12}\right) \xi_{1}^{2}+\left(a_{11} \partial_{1} a_{22}+a_{12} \partial_{1} a_{12}\right) \xi_{1} \xi_{2}+\left(a_{12} \partial_{1} a_{22}\right) \xi_{2}^{2} \\
=\frac{a_{11} a_{22}-a_{12}^{2}}{a_{22}^{2}}\left(a_{22} \partial_{1} a_{12}-a_{12} \partial_{1} a_{22}\right) \xi_{1}^{2}+\frac{1}{\beta_{2}} 0(|\xi|) .
\end{gathered}
$$

Due to the ellipticity condition (2.4), $a_{11} a_{22}-a_{12}^{2} \geq 0$. Hence $a_{22} \partial_{1} a_{12}-a_{12} \partial_{1} a_{22} \leq 0$ with $a_{22} \neq 0$ guarantees the positivity of $\mathcal{H}_{1}+\mathcal{H}_{2}$ for large $\beta_{2}$.

The proof is complete.

We observe that the condition (2.5) has a clear meaning of monotonicity of the speed of the propagation with respect to $x_{n}$ and is known and used in geophysics. In the most important case $n=3$ the additional conditions

$$
\begin{aligned}
& \frac{\partial_{1} a_{13}}{a_{13}} \leq \frac{\partial_{1} a_{33}}{a_{33}}, \\
& \frac{\partial_{1} a_{23}}{a_{23}} \leq \frac{\partial_{1} a_{33}}{a_{33}}, \\
& \frac{\partial_{2} a_{13}}{a_{13}} \leq \frac{\partial_{2} a_{33}}{a_{33}},
\end{aligned}
$$




$$
\frac{\partial_{2} a_{23}}{a_{23}} \leq \frac{\partial_{2} a_{33}}{a_{33}}
$$

are expected to be sufficient as well. We will give detailed proofs in the forthcoming paper. Whether these additional conditions are needed is an interesting open question.

\section{Proof of Theorem 1.3}

Let $\mathbf{u}(; 1)$ and $\mathbf{u}(; 2)$ satisfy $(1.6)$ corresponding to $R(; 1)$ and $R(; 2)$, respectively. Denote $\mathbf{u}=\mathbf{u}(; 2)-\mathbf{u}(; 1)$ and $\mathbf{F}=R(; 2)-R(; 1)=\left(f_{j k}\right), j, k=1, \ldots, 3$. By subtracting equations $(1.1)$ for $\mathbf{u}(; 1)$ from the equations for $\mathbf{u}(; 2)$ we yield

where

$$
\mathbf{A}_{R(; 2)} \mathbf{u}=\mathcal{A}(; \mathbf{u}(; 1)) \mathbf{F} \quad \text { on } \quad \Omega
$$

$$
\mathcal{A}(; \mathbf{u}(; 1)) \mathbf{F}=\sum_{j, k=1}^{3} f_{j k} \partial_{j} \partial_{k} \mathbf{u}(; 1) .
$$

Since the initial data for $\mathbf{u}(; 1), \mathbf{u}(; 2)$ are the same,

$$
\mathbf{u}=\partial_{t} \mathbf{u}=0 \quad \text { on } \quad \Omega \times\{0\} .
$$

Differentiating (3.1) in $t$ and using time-independence of the coefficients of the system, we get

$$
\mathbf{A}_{R(; 2)} \mathbf{U}=\mathcal{A}(; \mathbf{U}(; 1)) \mathbf{F} \quad \text { on } \quad \Omega
$$

where

$$
\mathbf{U}=\left(\begin{array}{c}
\partial_{t}^{2} \mathbf{u} \\
\partial_{t}^{3} \mathbf{u} \\
\partial_{t}^{4} \mathbf{u}
\end{array}\right) \quad \text { and } \quad \mathbf{U}(; 1)=\left(\begin{array}{c}
\partial_{t}^{2} \mathbf{u}(; 1) \\
\partial_{t}^{3} \mathbf{u}(; 1) \\
\partial_{t}^{4} \mathbf{u}(; 1)
\end{array}\right)
$$

Since $\partial G \times(-T, T)$ is noncharacteristic with respect to $\mathbf{A}_{R}$ we can uniquely solve the equation $\mathbf{A}_{R(; 2)} \mathbf{U}=0$ on $\partial G \times(-T, T)$ for $\partial_{\nu}^{2} \mathbf{U}$ in terms of $\mathbf{U}, \partial_{\nu} \mathbf{U}$, and their first and second tangential derivatives on $\partial G \times(-T, T)$. Moreover,

$$
\begin{gathered}
\left.\left\|\partial_{\nu}^{2} \mathbf{U}\right\|_{\left(\frac{1}{2}\right)}(\partial G \times(-T, T))\right) \\
\leq C\left(\|\mathbf{U}\|_{\left(\frac{5}{2}\right)}(\partial G \times(-T, T))+\left\|\partial_{\nu} \mathbf{U}\right\|_{\left(\frac{3}{2}\right)}(\partial G \times(-T, T))\right) \leq C F,
\end{gathered}
$$

due to definitions of $\mathbf{U}$ in (3.3) and of $F$ in (1.8).

By extension theorems for Sobolev spaces there exists $\mathbf{U}^{*} \in H_{(3)}(Q)$ such that

$$
\mathbf{U}^{*}=\mathbf{U}, \partial_{\nu} \mathbf{U}^{*}=\partial_{\nu} \mathbf{U}, \partial_{\nu}^{2} \mathbf{U}^{*}=\partial_{\nu}^{2} \mathbf{U} \quad \text { on } \quad \partial G \times(-T, T)
$$

and

due to the definition of $F$.

$$
\begin{aligned}
\left\|\mathbf{U}^{*}\right\|_{(3)}(\Omega) \leq C & \left(\|\mathbf{U}\|_{\left(\frac{5}{2}\right)}(\partial G \times(-T, T))+\left\|\partial_{\nu} \mathbf{U}\right\|_{\left(\frac{3}{2}\right)}(\partial G \times(-T, T))\right. \\
& \left.+\left\|\partial_{\nu}^{2} \mathbf{U}\right\|_{\left(\frac{1}{2}\right)}(\partial G \times(-T, T))\right) \leq C F
\end{aligned}
$$

We introduce $\mathbf{V}=\mathbf{U}-\mathbf{U}^{*}$. Due to (3.3),

$$
\mathbf{A}_{R(; 2)} \mathbf{V}=\mathcal{A}(; \mathbf{U}(; 1)) \mathbf{F}-\mathbf{A}_{R(; 2)} \mathbf{U}^{*} \quad \text { on } \quad \Omega,
$$

and due to $(3.5)$

$$
\mathbf{V}=\partial_{\nu} \mathbf{V}=\partial_{\nu}^{2} \mathbf{V}=0 \text {, on } \partial G \times(-T, T) .
$$

Since the system (3.7) is t-hyperbolic, by known energy estimates, (1.7), (3.8), and (3.6) give

$$
C^{-1}\left(E(0)-\|\mathbf{F}\|_{(1)}^{2}(G)-F^{2}\right) \leq E(t) \leq C\left(E(0)+\|\mathbf{F}\|_{(1)}^{2}(G)+F^{2}\right)
$$


where

$$
E(t)=E(t ; \mathbf{V})+E(t ; \nabla \mathbf{V}), E(t ; \mathbf{V})=\int_{G}\left(\left|\partial_{t} \mathbf{V}\right|^{2}+|\nabla \mathbf{V}|^{2}+|\mathbf{V}|^{2}\right)(, t)
$$

Here and below $\nabla$ is $\nabla_{x}$

On the other hand, by using the Carleman estimate of Theorem 1.1 and our choice of the weight function $\varphi$ we will bound the right side of (3.9) by a small fraction of $E(0)$ and given quantities.

To apply the Carleman estimate we need to cut off $\mathbf{V}$ near $t=T$ and $t=-T$. We first observe that from the definition and the conditions (1.12)

$$
1 \leq \varphi(x, 0), x \in G, \varphi(x, T)=\varphi(x,-T)<1 \quad \text { when } \quad x \in \bar{G} .
$$

So there exists a $\delta>\frac{1}{C}$ such that

$$
1-\delta<\varphi \quad \text { on } \quad G \times(0, \delta), \quad \varphi<1-2 \delta \quad \text { on } \quad G \times(T-2 \delta, T) .
$$

We now choose a smooth cut-off function $0 \leq \chi_{0}(t) \leq 1$ such that $\chi_{0}(t)=1$ for $-T+2 \delta<t<T-2 \delta$ and $\chi_{0}(t)=0$ for $|t|>T-\delta$. By the Leibniz' formula

$$
\mathbf{A}_{R(; 2)}\left(\chi_{0} \mathbf{V}\right)=\chi_{0} \mathcal{A}(; \mathbf{U}(; 1)) \mathbf{F}-\chi_{0} \mathbf{A}_{R(; 2)} \mathbf{U}^{*}+2 \rho\left(\partial_{t} \chi_{0}\right) \partial_{t} \mathbf{V}+\rho\left(\partial_{t}^{2} \chi_{0}\right) \mathbf{V}
$$

and hence

$$
\nabla \mathbf{A}_{R(; 2)}\left(\chi_{0} \mathbf{V}\right)=
$$

$$
\chi_{0} \nabla \mathcal{A}(; \mathbf{U}(; 1)) \mathbf{F}-\chi_{0} \nabla \mathbf{A}_{R(; 2)} \mathbf{U}^{*}+2 \rho\left(\partial_{t} \chi_{0}\right) \partial_{t} \nabla \mathbf{V}+\rho\left(\partial_{t}^{2} \chi_{0}\right) \nabla \mathbf{V} .
$$

Because of (3.8), $\chi_{0} \mathbf{V} \in H_{(3)}^{0}(\Omega)$, so we can apply the Carleman estimate (1.5) and (3.7) to get

$$
\begin{aligned}
\int_{G \times(0, T)} & \left(\left|\chi_{0} \mathbf{V}\right|^{2}+\left|\nabla_{x, t}\left(\chi_{0} \mathbf{V}\right)\right|^{2}+\left|\nabla_{x, t} \operatorname{div}\left(\chi_{0} \mathbf{V}\right)\right|^{2}+\left|\nabla_{x, t}\left(\chi_{0} \operatorname{curl} \mathbf{V}\right)\right|^{2}\right) e^{2 \tau \varphi} \\
\leq & C\left(\int_{\Omega}\left(|\mathbf{F}|^{2}+|\nabla \mathbf{F}|^{2}+\left|\mathbf{A}_{R(; 2)} \mathbf{U}^{*}\right|^{2}+\left|\nabla\left(\mathbf{A}_{R(; 2)} \mathbf{U}^{*}\right)\right|^{2}\right) e^{2 \tau \varphi}\right. \\
& \left.+\int_{G \times\{T-2 \delta<|t|<T\}}\left(|\mathbf{V}|^{2}+\left|\partial_{t} \mathbf{V}\right|^{2}+|\nabla \mathbf{V}|^{2}+\left|\partial_{t} \nabla \mathbf{V}\right|^{2}\right) e^{2 \tau \varphi}\right) .
\end{aligned}
$$

Using the known identity $\Delta \mathbf{V}=-\operatorname{curlcurl} \mathbf{V}+\nabla \operatorname{div} \mathbf{V}$ and the boundary conditions (3.8), from known elliptic estimates in the Dirichlet problem for the Laplace operator in $G$ we have

$$
\begin{gathered}
\int_{G}\left|\nabla^{2} \mathbf{V}\right|^{2} \leq C \int_{G}\left(|\nabla \operatorname{div} \mathbf{V}|^{2}+|\nabla \operatorname{curl} \mathbf{V}|^{2}\right), \\
\int_{G}\left|\partial_{t} \nabla \mathbf{V}\right|^{2} \leq C \int_{G}\left(\left|\partial_{t} \operatorname{div} \mathbf{V}\right|^{2}+\left|\partial_{t} \operatorname{curl} \mathbf{V}\right|^{2}\right)
\end{gathered}
$$

Hence integrating the energy bound $(3.9)$ over $(0, \delta)$ we conclude that

$$
\begin{gathered}
\delta E(0) \leq C\left(\int_{0}^{\delta} E(t) d t+\|\mathbf{F}\|_{(1)}^{2}(G)+F^{2}\right) \leq \\
C\left(\int_{G \times(0, \delta)}\left(|\mathbf{V}|^{2}+\left|\nabla_{x, t} \mathbf{V}\right|^{2}+\left|\nabla_{x, t} \operatorname{div} \mathbf{V}\right|^{2}+\left|\nabla_{x, t} \operatorname{curl} \mathbf{V}\right|^{2}+\|\mathbf{F}\|_{(1)}^{2}(G)+F^{2}\right) .\right.
\end{gathered}
$$

Similarly,

$$
\left.\int_{G \times\{T-2 \delta<|t|<T\}}\left(|\mathbf{V}|^{2}+\left|\partial_{t} \mathbf{V}\right|^{2}+|\nabla \mathbf{V}|^{2}+\left|\partial_{t} \nabla \mathbf{V}\right|^{2}\right) e^{2 \tau \varphi}\right) \leq
$$




$$
C\left(e^{2 \tau(1-2 \delta)}\left(E(0)+\|\mathbf{F}\|_{(1)}(G)\right)+C e^{2 \tau \Phi} F .\right.
$$

So from (3.11) and the remarks after (3.11), splitting the left side in (3.11) into two equal integrals, shrinking the integration domain to $G \times(0, \delta)$, using that $1-\delta<\varphi$ on $G \times(0, \delta)$ we finally yield

$$
\begin{gathered}
e^{2 \tau(1-\delta)} \delta E(0)+ \\
\int_{G \times(0, T)}\left(\chi_{0}|\mathbf{V}|^{2}+\left|\nabla_{x, t}\left(\chi_{0} \mathbf{V}\right)\right|^{2}\right) e^{2 \tau \varphi} \leq \\
C\left(\int_{\Omega}\left(|\mathbf{F}|^{2}+|\nabla \mathbf{F}|^{2}\right) e^{2 \tau \varphi}+e^{2 \tau \Phi} F^{2}+e^{2 \tau(1-2 \delta)}\left(E(0)+\int_{G}\left(|\mathbf{F}|^{2}+|\nabla \mathbf{F}|^{2}\right) .\right.\right.
\end{gathered}
$$

Choosing $\tau$ so large, that $e^{2 \tau(1-\delta)} \delta>2 C e^{2 \tau(1-2 \delta)}$ we will eliminate $E(0)$ from the right side. Since $\mathbf{U}=\mathbf{V}+\mathbf{U}^{*}$, using (3.6), from (3.13) we obtain

$$
\begin{gathered}
\int_{G \times(0, T)} \chi_{0}^{2}\left(|\mathbf{U}|^{2}+|\nabla \mathbf{U}|^{2}\right) \leq \\
C\left(e^{2 \tau \Phi} F^{2}+\int_{G}\left(\int_{-T}^{T} e^{2 \tau \varphi(x, t)} d t+e^{2 \tau(1-2 \delta)}\right)\left(|\mathbf{F}|^{2}+|\nabla \mathbf{F}|^{2}\right)(x)\right) d x
\end{gathered}
$$

Using (3.2) and (1.10) we get from (3.1) that $\rho\left(\partial_{t}^{2} \mathbf{u}, \partial_{t}^{3} \mathbf{u}\right)=\mathbf{M F}$ on $G \times\{0\}$. Therefore, using (1.10) we will have

$$
\begin{gathered}
\int_{G}\left(|\mathbf{F}|^{2}+|\nabla \mathbf{F}|^{2}\right) e^{2 s \varphi(, 0)} \leq C \int_{G} \sum\left|\partial_{t}^{\beta} \nabla^{k} \mathbf{u}(, 0)\right|^{2} e^{2 \tau \varphi(, 0)}= \\
\quad=-C \int_{0}^{T} \partial_{t}\left(\int_{G} \sum \chi_{0}^{2}\left|\partial_{t}^{\beta} \nabla^{k} \mathbf{u}\right|^{2} e^{2 \tau \varphi} d x\right) d t \\
\leq C \int_{\Omega} \chi_{0}^{2} \sum\left(\left|\partial_{t}^{\beta} \nabla^{k} \mathbf{u}\right|\left|\partial_{t}^{\beta+1} \nabla^{k} \mathbf{u}\right|+\tau\left|\partial_{t}^{\beta} \nabla^{k} \mathbf{u}\right|^{2}\right) e^{2 \tau \varphi} \\
\left.\quad+C \int_{G \times(T-2 \delta, T)} \chi_{0}\left|\partial_{t} \chi_{0}\right| \sum\left|\partial_{t}^{\beta} \nabla^{k} \mathbf{u}\right|^{2}\right) e^{2 \tau \varphi},
\end{gathered}
$$

where the sums are over $\beta=2,3, k=0,1$. Now the right side is less than

$$
\begin{aligned}
& C\left(\int_{\Omega} \tau \chi_{0}^{2}\left(|\mathbf{U}|^{2}+|\nabla \mathbf{U}|^{2}\right) e^{2 \tau \varphi}+\int_{G \times(T-2 \delta, T)}\left(|\mathbf{U}|^{2}+|\nabla \mathbf{U}|^{2}\right) e^{2 \tau \varphi}\right) \\
& \leq C\left(\int_{\Omega} \tau \chi_{0}^{2}\left(|\mathbf{U}|^{2}+|\nabla \mathbf{U}|^{2}\right) e^{2 \tau \varphi}+e^{2 \tau(1-2 \delta)}\left(\|\mathbf{F}\|_{(1)}^{2}(G)+F^{2}\right)\right),
\end{aligned}
$$

where we used equality $\mathbf{U}=\mathbf{U}^{*}+\mathbf{V}$ and (3.6), (3.9). From two previous bounds we conclude that

$$
\begin{gathered}
\int_{G}\left(|\mathbf{F}|^{2}+|\nabla \mathbf{F}|^{2}\right) e^{2 \tau \varphi(, 0)} \leq \\
C\left(\tau e^{2 \tau \Phi} F^{2}+\int_{G}\left(\int_{-T}^{T} e^{2 \tau \varphi(, t)} d t+e^{2 \tau(1-2 \delta)}\right)\left(|\mathbf{F}|^{2}+|\nabla \mathbf{F}|^{2}\right)\right) .
\end{gathered}
$$

Due to our choice of $\varphi, 1 \leq \varphi(, 0), \varphi(, t)-\varphi(, 0)<0$ when $t \neq 0$. Thus by the Lebesgue theorem, we have

$$
2 C\left(\int_{-T}^{T} e^{2 \tau \varphi(, t)} d t+e^{2 \tau(1-\delta)}\right) \leq e^{2 \tau \varphi(, 0)}
$$

uniformly on $G$ when $\tau>C$. Hence choosing and fixing such large $\tau$ (depending only on $C$ ) we eliminate the second term on the right side of (3.15).

The proof of Theorem 1.3 is now complete. 


\section{Conclusion}

So far there are no proofs of Lipschitz stability estimates with constants not depending on $R(2)$, but rather on their appropriate norms and constant $K$ in the pseudo-convexity condition (1.2). It is desirable and realistic to obtain Carleman estimate (1.5) without $\nabla$ on the right side and corresponding terms on the left side. Most likely it can be done by using Carleman estimates with two large parameters for general operators in Sobolev spaces of negative order or by using negative power of Laplacian in (1.5). Then one can obtain more natural stability estimates for inverse problems and to simplify their proofs. We plan to address these issues in near future. More challenging would be to obtain Carleman estimates, uniqueness of the continuation and coefficients identification results for the important system of transversally isotropic elasticity, where currently there are no results at all.

\section{Acknowledgments}

This work was supported in part by the Emylou Keith and Betty Dutcher Distinguished Professorship and the NSF grant DMS 07-07734.

\section{REFERENCES}

[1] H. Ammari, Y. Capdeboscq, H. Kang, A. Kozhemyak. Mathematical Models and Reconstruction Methods in Magneto-Acoustic Imaging, European J. Appl. Math., to appear.

[2] L. Hörmander Linear Partial Differential Operators, Springer-Verlag, 1963.

[3] O. Imanuvilov, V. Isakov, and M. Yamamoto, An inverse problem for the dynamical Lamé system with two sets of boundary data, Comm. Pure Appl. Math., 56 (2003), 1-17.

[4] V. Isakov, On the uniqueness of the continuation for a thermoelasticity system, SIAM J. Math. Anal., 33 (2001), 509-522.

[5] V. Isakov, Inverse Problems for Partial Differential Equations, Springer-Verlag, New York, 2006.

[6] V. Isakov, N. Kim, Carleman estimates with second large parameter for second order operators, Some application of Sobolev spaces to PDEs, International Math. Ser., Springer-Verlag, 10 (2008), 135-159

[7] V. Isakov, N. Kim, Carleman estimates with two large parameters for second order operators and applications to elasticity with residual stress, Applicationes Mathematicae, 3 (2008) (to appear).

[8] V. Isakov, G. Nakamura, J.-N. Wang, Uniqueness and stability in the Cauchy problem for the elasticity system with residual stress, Contemp. Math., AMS, 333 (2003), 99-113.

[9] V. Isakov, J.-N. Wang, M. Yamamoto, Uniqueness and stability of determining the residual stress by one measurement, Comm. Part. Diff. Equat., 23 (2007), 833-848.

[10] V. Isakov, J.-N. Wang, M. Yamamoto, An inverse problem for a dynamical Lame system with residual stress, SIAM J. Math. Anal., 39(2007), 1328-1343.

[11] A. Khaidarov, Carleman estimates and inverse problems for second order hyperbolic equations, Math. USSR Sbornik, 58 (1987), 267-277.

[12] I. Lasiecka, R. Triggiani, P.F. Yao, Inverse/observability estimates for second order hyperbolic equations with variable coeffcients, J. Math. Anal. Appl., 235 (2000), 13-57.

[13] C.-S. Man, Hartig's law and linear elasticity with initial stress, Inverse Problems, 14 (1998), 313-320. 